\title{
La integración efectiva del dispositivo móvil en la educación y en el aprendizaje
}

\section{Effective integration of the mobile device into education and learning}

\author{
María Soledad Ramírez-Montoya \\ Tecnológico de Monterrey, ITESM (México) \\ Francisco José García-Peñalvo \\ Universidad de Salamanca, USAL (España) \\ Tecnológico de Monterrey, ITESM (México) \\ Coordinadores del Monográfico
}

\section{Resumen}

Es un hecho que los dispositivos móviles, especialmente los teléfonos tipo smartphone, e Internet han cambiado sustancialmente las costumbres de la población, los hábitos sociales, las maneras de comunicación y, en definitiva, la forma de vida de las personas. Inevitablemente, aunque con diferentes barreras y con un tiempo quizás mayor del vaticinado por los estudios, la movilidad también ha llegado al contexto educativo, con diferente intensidad, penetración y uso en la formación formal y no formal, y de forma plena al ámbito del aprendizaje informal. El dispositivo móvil está llamado a revolucionar las prácticas educativas por aportar unos grados de libertad que otras tecnologías anteriores no ofrecían. La potencia de la conectividad ubicua y las posibilidades de contar con el acceso a las fuentes de conocimiento en un dispositivo de bolsillo, como el teléfono, o del tamaño de cuaderno en el caso de las tabletas, dotado además de una capacidad de procesamiento superior a la de ordenadores personales de hace unos pocos años, plantean escenarios disruptivos en las metodologías educativas con el apoyo de la tecnología y respaldan la formación en línea como una opción natural que se integra como complemento o como modelo formativo principal. No obstante, esta visión idílica tiene diferente grado de aceptación según el nivel educativo, el área disciplinar y la formalidad de la acción formativa. Por tanto, se necesita de estudios de investigación a gran escala que transmitan cuál es el grado de avance, uso efectivo, aceptación y penetración de las tecnologías móviles en la educación y el aprendizaje. Esta es la razón de ser de este número monográfico de la Revista Iberoamericana de Educación a Distancia (RIED), que se compone de 13 artículos seleccionados después de una rigurosa revisión por pares de las más de 35 propuestas recibidas.

Palabras clave: dispositivo móvil; educación; aprendizaje; mLearning; realidad aumentada; aprendizaje invertido; entornos personales de aprendizaje. 


\begin{abstract}
It is a real fact that mobile devices, specially smartphones, and Internet have substantially changed the customs of the population, the social habits, the communication media, and, ultimately, the way of life of people. Inevitably, although with different barriers and with more time that many studies and reports estimated, mobility has arrived to the educational context, with different intensity, penetration and usage in formal and non-formal education, and in a full way into the informal learning. Mobile device is called to revolutionize the educational practices due to it introduces some degrees of freedom that other previous technologies had not offered. The power of ubiquitous connectivity and the possibilities of having access to the knowledge sources in a pocket device, such as mobile phone, or with a size as a notebook, in the case of tablets, and also equipped with a processing capacity higher than that of personal computers a few years ago, pose disruptive scenarios in educational methodologies based on technology and support eLearning as a natural option both as a complement or as a main educational model. However, this idyllic vision has a different degree of acceptance according to the educational level, the disciplinary area and the formality of the formative action. Therefore, large-scale research studies are needed to convey the degree of advancement, effective use, acceptance and penetration of mobile technologies in education and learning. This is the reason for this special issue at the Revista Iberoamericana de Educacion a Distancia (RIED), which consists of 13 articles selected after a rigorous peer review of the more than 35 proposals received.
\end{abstract}

Keywords: mobile device; education; learning; mLearning; augmented reality; flipped learning; personal learning environments.

El acceso ubicuo a la información, tanto para consultarla como para generar nuevos datos en tiempo real, las posibilidades de conexión y comunicación, así como la diversidad de tipos de dispositivos (smartphones, tabletas, smartwatches, ebooks, etc.), que implican formas más ricas de interaccionar síncrona y asíncronamente, y todo ello unido a un constante incremento en la capacidad de proceso de estos dispositivos, convierte a los dispositivos móviles en auténticos agentes de cambio en los hábitos de las personas, en la manera de comunicarse y de interaccionar, bien entre ellas, bien con los servicios que se les ofertan desde empresas y administraciones. Además, los dispositivos móviles sirven para paliar ciertas barreras de inclusión debidas a discapacidades visuales, auditivas, motrices e intelectuales, mediante el amplio abanico de apps que ofrecen (Telefónica, 2012).

El dominio educativo se ve muy afectado con los avances tecnológicos (GarcíaPeñalvo y Colomo-Palacios, 2015; García-Peñalvo y Llamas Nistal, 2017), abriendo posibilidades, escenarios y retos, en los que desde tiempo el mobile learning o mLearning (Alonso de Castro, 2014; Briz-Ponce, Juanes-Méndez y García-Peñalvo, 2016; Sánchez-Prieto, Olmos-Migueláñez y García-Peñalvo, 2013, 2014) ha sido una constante promesa (Johnson, Adams y Cummins, 2012; Johnson et al., 2013) que, actualmente, ya es una realidad inevitable, de forma que eLearning, mLearning y bLearning, pasan de ser una opción a convertirse en un requisito de supervivencia 
para las instituciones educativas, con un plazo de adopción inmediato según el último informe NMC Horizon Report: 2017 Higher Education Edition (Adams Becker et al., 2017).

Múltiples informes y estudios avalan la creciente importancia y penetración de los dispositivos móviles en la sociedad, con especial atención a los smartphones. Según International Telecommunication Union (2016) en los países desarrollados el $95 \%$ de las personas adultas poseen un teléfono móvil, mientras que en los países en desarrollo la penetración es del 90\%. España es líder en número de smartphones, donde un $80 \%$ de los españoles dispone de uno, frente al $78 \%$ que tiene un PC, ya sea portátil o de sobremesa. Este porcentaje aumenta entre los menores, donde el 98\% de los jóvenes entre los 10 y los 14 años tiene un teléfono inteligente con acceso a Internet (Ditrendia, 2016). En 2016 StatCounter (https://goo.gl/X380Fk) informó que el 51,3\% del acceso a la Web se había realizado mediante móviles y tabletas, sobrepasando por primera vez al acceso desde el ordenador de sobremesa. El proceso de producción de contenidos para estos dispositivos también representa un reto para lograr aprendizaje móvil (Glasserman y Ramírez-Montoya, 2012) y, sin duda, la tecnología educativa tiene ante sí un área de oportunidad para ampliar sus ámbitos de aplicación (Ramírez-Montoya y Burgos, 2012). Según el Cisco Visual Networking Index, el tráfico de Internet debido a los smartphones superará al de los PC en 2020, y en conjunto el tráfico de las redes wifi y de los dispositivos móviles supondrá dos tercios del total del tráfico en ese año (CISCO, 2016).

En un estudio realizado en España sobre los dispositivos tecnológicos más usados, con 183 respuestas válidas, el smartphone fue el más elegido con independencia de género y edad, siendo el grupo de edad entre 30 y 60 el más activo, con una media cercana al uso diario con una desviación típica de 0,49 . Si esta pregunta se traslada al dominio educativo, la primera opción del grupo entre 30 y 60 es el PC, seguido del laptop y del smartphone; el grupo de menores de 30 elige el laptop como primer dispositivo, el PC como segundo y la tableta como tercero; por último, el grupo de mayores de 60 también se decanta por el PC como el dispositivo más usado para fines de aprendizaje, seguido con una diferencia mínima por la tableta y ya en tercer lugar destaca el laptop (Fonseca Escudero, Conde González y García-Peñalvo, 2017).

En otro estudio a gran escala que se realizó en México, se integró el uso de dispositivos móviles en ambientes multimodales, bLearning y de educación a distancia, se analizó el uso en más de 3000 estudiantes universitarios (RamírezMontoya, 2009), se estudiaron los componentes pedagógicos, tecnológicos y de diseño en la producción de materiales para dispositivos móviles (Contreras, Herrera y Ramírez-Montoya, 2009; Sandoval, García y Ramírez-Montoya, 2012), el potencial para apoyar el desarrollo de habilidades cognitivas con recursos de mLearning (Ramos, Herrera-Bernal y Ramírez-Montoya, 2010), el desarrollo de competencias aplicadas por los estudiantes para el uso de dispositivos mLearning (HerreraBernal, Ramírez-Hernández y Ramírez-Montoya, 2016), así como las características predominantes en la generación Net que favorecen el aprendizaje al usar dispositivos 
móviles (Padrón Moctezuma, Ochoa-Vásquez y Ramírez-Montoya, 2016). A través de estos estudios se evidenciaron las dificultades que aún se tienen, pese al avance de las tecnologías, en cuanto a los tiempos, costos y aplicaciones para una integración eficaz en los ámbitos formativos.

En este sentido, hablar del mero uso de dispositivos en el aula como sinónimo de mLearning ya no es posible hoy en día, como lo fue no hace demasiados años, ahora se debe demandar que estos dispositivos vengan acompañados de un enfoque pedagógico y/o de un marco educativo que redunde en alguna mejora del proceso formativo. Es decir, se debe superar el estado actual, todavía embrionario, en el que abundan las iniciativas aisladas, sin continuidad y poco exitosas que no traen consigo un cambio metodológico real (Fernández Rodrigo, 2016; Sánchez-Prieto, Olmos-Migueláñez, García-Peñalvo y Torrecilla-Sánchez, 2016) y desaprovechan el potencial para la innovación que ofrece el mLearning (Sung, Chang y Liu, 2016), incluso, en los mismos procesos para formar en ambientes presenciales (RamírezMontoya, 2017) y a distancia, donde, por su misma naturaleza, tienen un amplio margen de operación y apoyo (Glasserman y Ramírez-Montoya, 2015).

Esta es la razón de ser de este número monográfico de la Revista Iberoamericana de Educación a Distancia (RIED), para aportar estudios de investigación a gran escala que transmitan cuál es el grado de avance, uso efectivo, aceptación y penetración de las tecnologías móviles en la educación y el aprendizaje.

\section{CONTENIDOS DEL NÚMERO MONOGRÁFICO}

El monográfico "La integración efectiva del dispositivo móvil en la educación y en el aprendizaje" se compone de 13 artículos seleccionados (Agila-Palacios, RamírezMontoya, García-Valcárcel y Samaniego-Franco, 2017; Berns, Palomo-Duarte, IslaMontes, Dodero y Torre, 2017; Cabero Almenara, Fernández Robles y Marín Díaz, 2017; Gutiérrez Esparza, Margain Fuentes, Canul Reich y Ramírez del Real, 2017; Humanante-Ramos, García-Peñalvo y Conde-González, 2017; Joo-Nagata, Martínez Abad y García-Bermejo Giner, 2017; Marçal, Castro Andrade, Viana, 2017; Martín y Tourón, 2017; Martín-Ramos, Ramos Silva y Pereira da Silva, 2017; Mateus, AranRamspott y Masanet, 2017; Mülbert y Cybis Pereira, 2017; Redondo Domínguez, Fonseca Escudero, Sánchez Riera y Navarro Delgado, 2017; Sánchez-Prieto, OlmosMigueláñez y García-Peñalvo, 2017b) de las más de 35 propuestas recibidas.

En la figura 1 se muestra un mapa conceptual en el que se categorizan los artículos incluidos en el monográfico atendiendo a 7 características o dimensiones, que son:

1. Dispositivo móvil.

2. Tipo de contribución.

3. Método.

4. Instrumento.

5. Nivel educativo. 
6. Técnica/herramienta.

7. Dominio de aplicación.

\section{Dispositivo móvil}

La mayoría de los artículos de este monográfico hablan de dispositivo móvil en general, aunque haciendo referencia a una generalización del smartphone y la tableta. Tres artículos se refieren específicamente a un smartphone, dos de ellos se basan en apps concretas desarrolladas en el contexto de la contribución (Berns et al., 2017; Joo-Nagata, Martínez Abad et al., 2017) mientras que en el tercero utilizan el móvil como herramienta de simulación en combinación con otras herramientas (Martín-Ramos et al., 2017). Solo uno de los artículos se refiere específicamente a las tabletas como herramienta en entornos de aprendizaje a distancia (Agila-Palacios et al., 2017).

\section{Tipo de contribución}

Son muchos los tipos de artículos que se pueden publicar en una revista. En el caso de este monográfico ocho de las contribuciones se pueden considerar como artículos de investigación, tres como experiencias de innovación educativa (Marçal et al., 2017; Martín-Ramos et al., 2017; Redondo Domínguez et al., 2017). Las otras dos contribuciones son artículos de revisión (Grant y Booth, 2009) que aplican un protocolo específico para sistematizar la búsqueda delas contribuciones que satisfacen las preguntas de investigación que se plantean los autores (Fink, 1998). Este tipo de contribuciones aportan un alto valor en el campo disciplinar en el que se aplican y se consideran investigaciones científicas en sí mismas, con métodos prefigurados y un ensamblaje de los estudios originales, que sintetizan los resultados de estos (Gisbert y Bonfill, 2004). De las dos contribuciones presentes en el monográfico Humante et al. (2017) utilizan una aproximación de revisión sistemática de literatura, mientras Mateus et al. (2017) se acercan más al concepto de mapeo sistemático.

\section{Método}

Los métodos de investigación utilizados en los diferentes artículos son bastante variados con un predominio de los métodos cuantitativos, ya sea de forma única (Cabero Almenara et al., 2017; Joo-Nagata, Martínez Abad, et al., 2017; SánchezPrieto et al., 2017b) o de forma mixta con alguna propuesta cualitativa (Martín y Tourón, 2017; Redondo Domínguez et al., 2017). Por su parte, Mülbert y Cybis Pereira (2017) aplican DBR (Design-Based Research) (Anderson y Shattuck, 2012), que es un paradigma de investigación que se desarrolla en contextos reales y se 
centra en el diseño y ensayo de intervenciones y prácticas educativas significativas, adoptando, en este caso, métodos mixtos.

Tres artículos utilizan alguna forma de minería de datos, Berns et al. (2017) de una manera muy básica para registrar la actividad en la app de agenda colaborativa que proponen; Agila et al. (2017) aplican clusterización, a través de la metodología CRISP-DM (Cross Industry Standard Process for Data Mining) (Chapman et al., 2000), tanto a la participación en el entorno virtual de aprendizaje como en la interacción con la tableta; y Gutiérrez Esparza et al. (2017) utilizan el modelo computacional SocialMining (Gutiérrez Esparza, Padilla Díaz, Canul-Reich, DeLuna y Ponce, 2016) para clasificar comentarios en Twitter.

Los dos artículos de revisión (Humanante-Ramos et al., 2017; Mateus et al., 2017) van a aplicar métodos de SLR (Systematic Literature Review) (Kitchenham y Charters, 2007).

Por último, Marçal et al. (2017) emplean el método del caso de estudio para presentar su experiencia de innovación.

\section{Instrumento}

En cuanto a los instrumentos, los artículos de revisión (Humanante-Ramos et al., 2017; Mateus et al., 2017) utilizan protocolos de revisión para sistematizar el proceso.

Varios trabajos aplican diversas técnicas de analítica de datos (Agila-Palacios et al., 2017; Berns et al., 2017; Gutiérrez Esparza et al., 2017; Martín y Tourón, 2017).

Aunque el instrumento más utilizado es algún tipo de cuestionario (Joo-Nagata, Martínez Abad et al., 2017; Martín y Tourón, 2017; Redondo Domínguez et al., 2017). De una forma más específica, Cabero Almenara et al. (2017) utilizan el Instructional Material Motivational Survey (IMMS) (Keller, 2010) para estudiar la motivación de los estudiantes, así como de las dimensiones que lo conforman: atención, renovación, relevancia y satisfacción, por utilizar apuntes enriquecidos con objetos de realidad aumentada; Sánchez-Prieto et al. (2017b), avanzando en el trabajo realizado previamente (Sánchez-Prieto, Olmos-Migueláñez y García-Peñalvo, 2016a, 2016b, 2016c, 2017a, 2017c), utilizan un modelo Technology Acceptance Model (TAM) (Davis, 1989) que incluye dos constructos adicionales, el entretenimiento percibido (Davis, Bagozzi y Warshaw, 1992) y la resistencia al cambio (Lewin, 1947); Marçal et al. (2017) utilizan un cuestionario dividido en tres partes, la primera de ellas se basa en System Usability Scale (SUS) (Brooke, 1996, 2013) para obtener información sobre la facilidad de uso, la segunda de ellas está basada en TAM para identificar la utilidad percibida por los estudiantes, y, por último, la tercera parte se compone de preguntas abiertas para recoger aspectos positivos, negativos y sugerencias por parte de los estudiantes. 
M. Ramírez-Montoya; F. García-Peñalvo

LA INTEGRACIÓN EFECTIVA DEL DISPOSITIVO MÓVIL EN LA EDUCACIÓN Y EN EL APRENDIZAJE

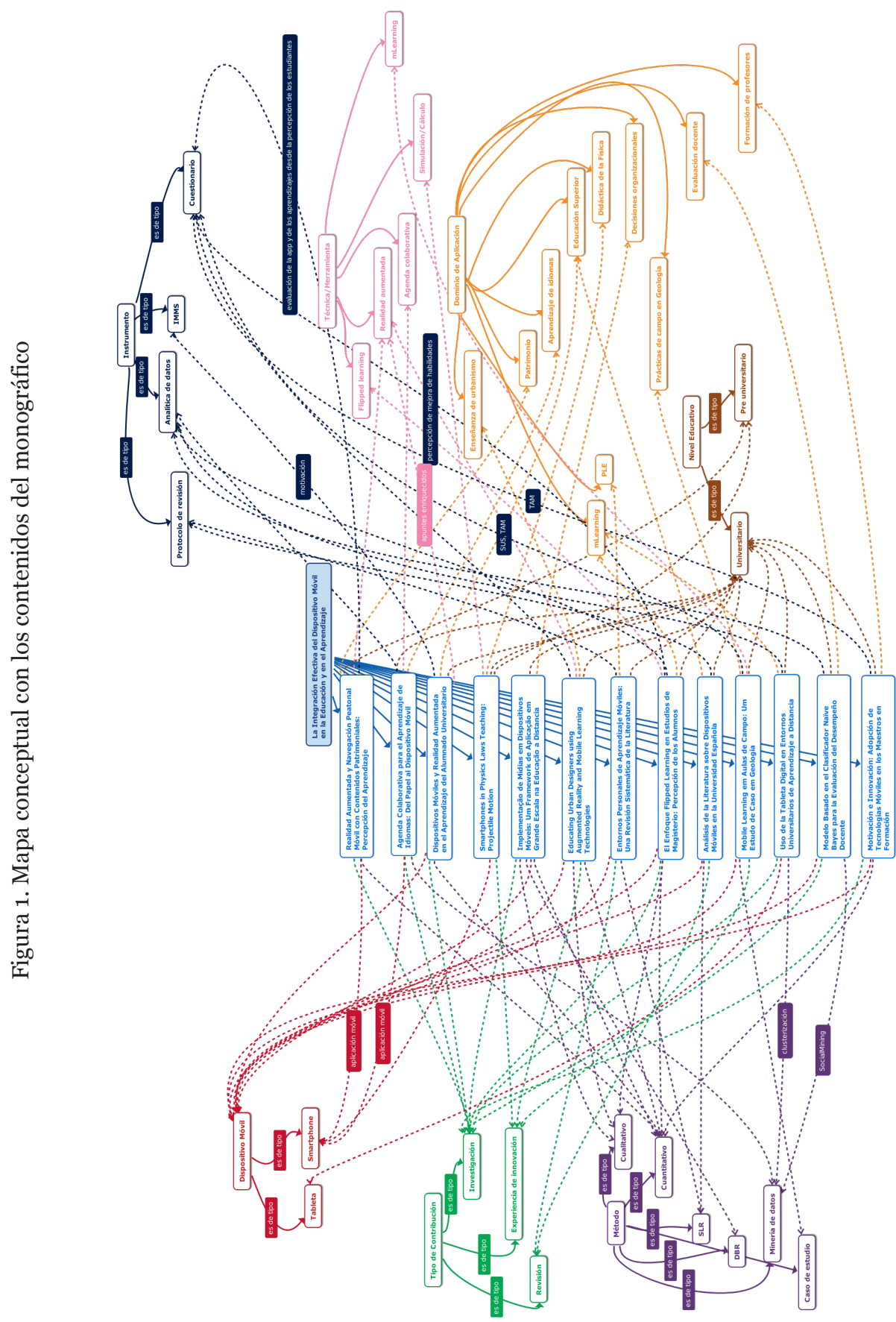




\section{Nivel educativo}

Esta dimensión establece hacia qué nivel educativo se han orientado las contribuciones. Finalmente, solo se han detectado dos opciones, el nivel universitario, que es el mayoritario, y el preuniversitario, representado este por solo dos contribuciones (Joo-Nagata, Martínez Abad et al., 2017; Martín-Ramos et al., 2017), aunque hay que destacar que la contribución de Martín-Ramos et al., se aplica tanto a nivel universitario como preuniversitario.

\section{Técnica/Herramienta}

Con esta dimensión se pretende recoger el dominio pedagógico que acompaña a la investigación o a la experiencia basada en dispositivos móviles (no es aplicable al caso de los artículos de revisión).

Tres contribuciones utilizan la Realidad Aumentada como propuesta pedagógica con uso de dispositivos móviles. El uso de la Realidad Aumentada con fines educativos se está potenciando significativamente (Cabero Almenara y Barroso, 2016; Chen, Liu, Cheng y Huang, 2017) gracias a la facilidad de acceder a sus prestaciones a través de los dispositivos móviles, esto es, combinar información digital y física en tiempo real con smartphones y tabletas para crear nuevas realidades. Además, la movilidad le otorga a la Realidad Aumentada la capacidad de crear actividades educativas sin las limitaciones de equipos ni de lugares fijos, en las que la localización espacial tiene una especial relevancia y utilidad (Aurelia, Raj y Saleh, 2014; Joo-Nagata, García-Bermejo Giner y Martínez Abad, 2017; Joo-Nagata, García-Bermejo Giner y Muñoz Rodríguez, 2015; Joo-Nagata, Martínez Abad, García-Bermejo Giner y García-Peñalvo, 2017) porque posibilita una interacción tangible en entornos reales mediante marcadores y experiencias colaborativas en tiempo real (Sánchez, Redondo y Fonseca, 2012). De forma específica en este monográfico, Cabero Almenara et al. (2017) proponen el uso de apuntes enriquecidos con marcados de realidad aumentada; Joo-Nagata et al. (2017) utilizan un sistema de localización peatonal para aportar más información sobre el patrimonio cultural en una ruta establecida; y Redondo Domínguez et al. (2017) diseñan ejercicios prácticos sobre la casuística de un proyecto real, en los que los estudiantes deben diseñar una escultura para una plaza pública en función de los parámetros del entorno, para ello usarán plataformas móviles de realidad aumentada, y así enfatizar un aprendizaje colaborativo mediante el estudio de las propuestas del resto de estudiantes in situ.

El aprendizaje invertido (flipped learning) (Observatorio de Innovación Tecnológica del Tecnológico de Monterrey, 2014) intenta dar un giro al método tradicional de instrucción directa, para ello propone impartir la parte teórica fuera del tiempo de la clase, generalmente mediante vídeos, y aprovechar el tiempo en el aula para realizar actividades de aprendizaje más significativas (Fulton, 2014; García-Peñalvo, Fidalgo-Blanco, Sein-Echaluce Lacleta y Conde-González, 2016; 
Ramírez-Montoya y Ramírez-Hernández, 2016; Sein-Echaluce Lacleta, Fidalgo Blanco y García-Peñalvo, 2015; Tourón y Santiago, 2015). En este monográfico, Martín y Tourón (2017) proponen que el mLearning se aplique en el desarrollo de una experiencia de aprendizaje invertido, incorporando metodologías activas durante las clases presenciales en las que se utilizan dispositivos móviles para combinar el entorno formal e informal (García-Peñalvo y Griffiths, 2014, 2015; Griffiths y García-Peñalvo, 2016).

El mLearning está implícito en todos los artículos del monográfico, pero como técnica principal aparece en la aportación de Marçal et al. (2017) al utilizarlo como estrategia educativa en las prácticas de campo, con idea de mejorar el aprendizaje gracias a la interacción que se produce entre los estudiantes y sus profesores, la reflexión propia de las actividades activas y la facilidad de consultar la información necesaria en tiempo real (Lai y Hwang, 2014).

Otras técnicas y herramientas que se han utilizado para darle un sentido pedagógico al uso de los dispositivos móviles en el aula son las simulaciones (MartínRamos et al., 2017) y la agenda colaborativa (Berns et al., 2017).

\section{Dominio de aplicación}

La última de las dimensiones que se ha tenido en cuenta para clasificar las contribuciones de este monográfico ha sido el campo en el que se han aplicado las diferentes experiencias. Los dominios de aplicación han sido prácticamente tantos como contribuciones, como se puede apreciar en los siguientes párrafos.

La enseñanza del urbanismo y el patrimonio (Joo-Nagata, Martínez Abad et al., 2017; Redondo Domínguez et al., 2017) con la realidad aumentada como principal soporte tecnológico.

El aprendizaje de idiomas es uno de los nichos de mercado de las aplicaciones móviles, con importantes apps que copan el interés de millones de usuarios, como por ejemplo ABA English o Duolingo por citar solo algunos casos de éxito. Este dominio es, por tanto, un área de investigación muy atractiva (Burston, 2015). Berns et al. (2017) presentan Terminkalender, una app multiusuario específicamente diseñada para facilitar el aprendizaje colaborativo de alemán a través de dispositivos móviles, y que supone una continuidad en esta línea de investigación de estos autores (Berns, Isla-Montes, Palomo-Duarte y Dodero, 2016; Berns, Palomo-Duarte, Dodero, Ruiz Ladrón y Calderón Márquez, 2015; Palomo-Duarte, Berns, Cejas, Dodero y Caballero, 2016; Ruiz-Rube, Miguel Mota, Person, Berns y Dodero Beardo, 2016).

Las decisiones estratégicas en relación a la Educación Superior (Cabero Almenara et al., 2017; Mateus et al., 2017; Mülbert y Cybis Pereira, 2017), el uso del mLearning como aproximación docente (Martín y Tourón, 2017) o la evaluación del desempeño docente (Gutiérrez Esparza et al., 2017) constituyen otro de los dominios de interés de las contribuciones del monográfico. 
El uso didáctico de los dispositivos móviles en la enseñanza de la Física (MartínRamos et al., 2017) o en las prácticas de campo de Geología (Marçal et al., 2017) es otro ejemplo de dominio de aplicación.

Humante et al. (2017) abordan la evolución del concepto de Personal Learning Environment (PLE) (Attwell, 2007; Conde et al., 2014; Wilson et al., 2007) al de mobile PLE (mPLE) (Conde, García-Peñalvo, Alier, \& Piguillem, 2013; HumanteRamos, García-Peñalvo y Conde-González, 2016) mediante una revisión sistemática de literatura.

Por último, la formación del futuro profesorado en el uso de los dispositivos móviles como herramienta educativa es fundamental para la consolidación del mLearning (Sánchez-Prieto et al., 2017b).

\section{CONCLUSIONES}

Los dispositivos móviles tienen una presencia diaria y continua en la vida de las personas. Se han convertido de facto en el complemento tecnológico por excelencia, con el que se realizan cada vez más tipos de tareas y en el que volcamos una mayor parte de nuestro perfil digital. El smartphone tiene presencia tanto en el mundo desarrollado, como en los países emergentes y en vías de desarrollo, véase el crecimiento de la penetración en África, e incluso en las zonas más remotas del planeta, como, por ejemplo, la región de la Patagonia Argentina, donde este dispositivo se convierte en una de las inversiones tecnológicas preferidas de sus habitantes.

No es de extrañar que, si los dispositivos móviles se han convertido en una extensión de los individuos, estos se usen en una actividad tan habitual, importante y necesaria como es el aprendizaje. Desde una perspectiva informal, estos dispositivos móviles, especialmente los smartphones, ya generan tráfico web que los dispositivos de sobremesa, lo que implica una consulta continuada a diversas fuentes de información. Desde un punto de vista formal, en las aulas universitarias smartphones, tabletas y portátiles ya forman parte habitual de la decoración de un aula universitaria, en la que los estudiantes consultan el campus virtual y toman notas mientras el profesor explica; uso este que se combina con grupos informales, usando aplicaciones como Whatsapp (Karapanos, Teixeira y Gouveia, 2016), en los que los estudiantes intercambian más información entre ellos que en los foros establecidos en los canales oficiales. Este uso se extiende hacia diferentes niveles educativos, donde, por ejemplo, la educación básica ha encontrado apoyo para desarrollar competencias de apropiación tecnológica a través de tabletas digitales (Escobar, Glasserman y Ramírez-Montoya, 2015).

Si se entendiera por mLearning este uso cotidiano del dispositivo móvil con algún fin educativo, se podría decir que estaríamos en plena madurez del concepto. Sin embargo, la concepción que se tiene hoy en día del mLearning dista mucho del simple manejo del dispositivo, y requiere para su uso efectivo de que este dispositivo 
se encuentre inmerso en un marco pedagógico definido, por actividades definidas instruccionalmente en las que el Smartphone, o la tableta, es algo más que un mero navegador de información.

En este monográfico se han presentado interesantes y exitosos casos que así lo entienden y lo desarrollan. Sin embargo, la escalabilidad del caso de éxito del mLearning a la práctica generalizada es el verdadero reto al que se enfrentan hoy las instancias educativas para lograr esa adopción efectiva inmediata que se reclama por parte de los diversos informes sobre la Sociedad del Conocimiento y el uso de las tecnologías en el ámbito educativo.

\section{AGRADECIMIENTOS}

Los editores invitados de este monográfico no quieren dejar de mostrar su más sincero agradecimiento a todas las personas que han hecho posible llegar al final de un proceso largo, intenso y muy satisfactorio. En primer lugar, a todos los autores que han enviado sus contribuciones (más de una treintena), independientemente de su éxito, porque son imprescindibles para tener una selección de calidad contrastada como la que aquí se tiene. Después, a todos los revisores científicos, quienes con su exigente juicio y con total independencia de los editores invitados, han establecido el nivel de aceptación y, por tanto, la calidad del monográfico. Por último, y de una manera muy especial al editor en jefe de la Revista Iberoamericana de Educación a Distancia (RIED), el Dr. Lorenzo García Areitio, por su constante apoyo y compromiso con este proceso.

El uso de los dispositivos móviles en los procesos de enseñanza y aprendizaje están relacionados, fundamentalmente, con dos proyectos compartidos por los editores invitados, el proyecto DEFINES (Ref. TIN2016-80172-R), financiado por el Ministerio de Economía y Competitividad del Gobierno de España, y el proyecto Laboratorio BiNacional para la Gestión Inteligente de la Sustentabilidad Energética y Formación Tecnológica (Ref. 266632), financiado por la Secretaría de Energía y el Consejo Nacional de Ciencia y Tecnología, CONACYT (Fondo SENER-CONACYT, 2016-2019) de México.

\section{REFERENCIAS BIBLIOGRÁFICAS}

Adams Becker, S., Cummins, M., Davis, A., Freeman, A., Hall Giesinger, C., y Ananthanarayanan, V. (2017). NMC Horizon Report: 2017 Higher Education Edition. Austin, Texas: The New Media Consortium.
Alonso de Castro, M. G. (2014). Educational projects based on mobile learning. Education in the Knowledge Society, 15(1), 10-19.

Anderson, T., y Shattuck, J. (2012). Design-Based Research: A decade of progress in education research? 
Educational Researcher, 41(1), 16-25. doi:10.3102/0013189X11428813

Attwell, G. (2007). The Personal Learning Environments - The future of eLearning? eLearning Papers, 2(1).

Aurelia, S., Raj, M. D., y Saleh, O. (2014). Mobile Augmented Reality and Location Based Service. En N. Mastorakis, K. Psarris, G. Vachtsevanos, P. Dondon, V. Mladenov, A. Bulucea, I. Rudas y O. Martin (Eds.), Advances in Information Science and Applications. Proceedings of the 18th International Conference on Computers (part of CSCC '14), Santorini Island, Greece July 17-21, 2014 (Vol. II, pp. 551-558). Estados Unidos: INASE.

Berns, A., Isla-Montes, J. L., Palomo-Duarte, M. y Dodero, J. M. (2016). Motivation, students' needs and learning outcomes: A hybrid game-based app for enhanced language learning. SpringerPlus, 5(1), 1305. doi:10.1186/s40064-016-2971-1

Berns, A., Palomo-Duarte, M., Dodero, J. M., Ruiz Ladrón, J. M., y Calderón Márquez, A. (2015). Mobile apps to support and assess foreign language learning. In F. Helm, L. Bradley, M. Guarda y S. Thouësny (Eds.), Critical CALL - Proceedings of the 2015 EUROCALL Conference (22nd, Padova, Italy, August 26-29, 2015) (pp. 51-56): Research-publishing.net.

Briz-Ponce, L., Juanes-Méndez, J. A., y García-Peñalvo, F. J. (Eds.). (2016). Handbook of Research on Mobile Devices and Applications in Higher Education Settings. Hershey, PA: IGI Global.

Brooke, J. (1996). SUS - A quick and dirty usability scale. Usability evaluation in industry, 189(194), 4-7.

Brooke, J. (2013). SUS: A Retrospective. Journal of Usability Studies, 8(2), 29-40.
Burston, J. (2015). Twenty years of MALL project implementation: A meta-analysis of learning outcomes. ReCALL, 27(1), 4-20. doi:10.1017/So958344014000159

Cabero Almenara, J., y Barroso, J. (2016). The educational possibilities of Augmented Reality. NAER. New Approaches in Educational Research, 5(1), 44-50. doi: 10.7821/naer.2016.1.140.

Chapman, P., Clinton, J., Kerber, R., Khabaza, T., Reinartz, T., Shearer, C., y Wirth, R. (2000). CRISP-DM 1.o. Step-by-step data mining guide (CRISPMWP-1104). Recuperado de https://www.themodeling-agency.com/crisp-dm.pdf

Chen, P., Liu, X., Cheng, W., y Huang, R. (2017). A review of using Augmented Reality in Education from 2011 to 2016. En E. Popescu, Kinshuk, M. K. Khribi, R. Huang, M. Jemni, N.-S. Chen, y D. G. Sampson (Eds.), Innovations in Smart Learning. (13-18). Singapore: Springer.

CISCO. (2016). Cisco Visual Networking Index: Forecast and Methodology, 20152020. Recuperado de https://goo.gl/ biWofs

Conde, M.Á., García-Peñalvo, F.J., Alier, M., y Piguillem, J. (2013). The implementation, deployment and evaluation of a Mobile Personal Learning Environment. Journal of Universal Computer Science, 19(7), 854-872. doi:10.3217/jucs-019-07-0854

Conde, M. Á., García-Peñalvo, F. J., Rodríguez-Conde, M. J., Alier, M., Casany, M. J., y Piguillem, J. (2014). An evolving Learning Management System for new educational environments using 2.0 tools. Interactive Learning Environments, 22(2), 188-204. doi: 10.1080/10494820.2012.745433.

Contreras, J., Herrera, J. A., y RamírezMontoya, M. S. (2009). Elementos 
instruccionales para el diseño y la producción de materiales educativos móviles. Apertura Revista de innovación educativa, 5(11).

Davis, F. D. (1989). Perceived Usefulness, Perceived Ease of Use, and User Acceptance of Information Technology. MIS Quarterly, 13(3), 319-340.

Davis, F. D., Bagozzi, R. P., y Warshaw, P. R. (1992). Extrinsic and intrinsic motivation to use computers in the Workplace. Journal of Applied Social Psychology, 22(14), 1111-1132. doi: 10.1111/j.15591816.1992.tboo945.x.

Ditrendia. (2016). Informe ditrendia 2016: Mobile en España y en el Mundo. Recuperado de http://www.amic.media/ media/files/file 352 1050.pdf

Escobar, J. V., Glasserman. L. D., y RamírezMontoya, M. S. (2015). Apropiación tecnológica con pizarrón interactivo y tabletas digitales en profesores de educación básica. EDUTEC, Revista Electrónica de Tecnología Educativa, 53, 1-15.

Fernández Rodrigo, L. (2016). El uso didáctico y metodológico de las tabletas digitales en aulas de educación primaria y secundaria de Cataluña. Pixel-Bit. Revista de Medios y Educación, 48, 9-25.

Fink, A. (1998). Conducting literature research reviews: from paper to the Internet. Thousand Oaks, CA: Sage.

Fonseca Escudero, D., Conde González, M. Á., \& García-Peñalvo, F. J. (2017). Information society skills: Is knowledge accessible for all? Universal Access in the Information Society, En Prensa.

Fulton, K. P. (2014). Time for learning: Top 10 reasons why flipping the classroom can change education. California, USA: Corwin Press.
García-Peñalvo, F. J., y Colomo-Palacios, R. (2015). Innovative teaching methods in Engineering. International Journal of Engineering Education (IJEE), 31(3), 689-693.

García-Peñalvo, F. J., Fidalgo-Blanco, Á., Sein-Echaluce Lacleta, M., y CondeGonzález, M. Á. (2016). Cooperative Micro Flip Teaching. En P. Zaphiris y I. Ioannou (Eds.), Learning and Collaboration Technologies. Procedings of Third International Conference, LCT 2016, Held as Part of HCI International 2016, Toronto, ON, Canada, (pp. 1424). Switzerland: Springer International Publishing.

García-Peñalvo, F. J., y Griffiths, D. (2014). Transferring knowledge and experiences from informal to formal learning contexts. En F. J. García-Peñalvo (Ed.), Proceedings of the Second International Conference on Technological Ecosystems for Enhancing Multiculturality (TEEM'14) (pp. 569572). New York, USA: ACM.

García-Peñalvo, F. J., y Griffiths, D. (2015). Rethinking informal learning. En G. R. Alves y M. C. Felgueiras (Eds.), Proceedings of the Third International Conference on Technological Ecosystems for Enhancing Multiculturality (TEEM'15) (Porto, Portugal, October 7-9, 2015) (pp. 457-459). New York, USA: ACM.

García-Peñalvo, F. J., y Llamas Nistal, M. (2017). The Engineering Behind the Technological-Based Educational Innovation. International Journal of Engineering Education (IJEE), 33(2B), 763-767.

Gisbert, J., y Bonfill, X. (2004). ¿Cómo realizar, evaluar y utilizar revisiones sistemáticas y metaanálisis? Gastroenterología y 
Hepatología, 27(3), 129-149. doi:10.1016/ So210-5705(03)79110-9

Glasserman, L. D., y Ramírez-Montoya, M. S. (2012). Procesos de formación en la producción y uso de recursos educativos abiertos y móviles: percepciones de autores y usuarios. En M. S. RamírezMontoya y J. V. Burgos (Eds.), Recursos educativos abiertos y móviles para la formación de investigadores: Investigaciones y experiencias prácticas (85-97). México: Lulú editorial digital.

Glasserman, L. D., y Ramírez-Montoya, M. S. (2015). Formación de investigadores educativos mediante el diseño de recursos educativos abiertos y móviles. Revista de Investigación Educativa de la Escuela de Graduados en Educación, 5(10), 36-42.

Grant, M. J., y Booth, A. (2009). A typology of reviews: an analysis of 14 review types and associated methodologies. Health Information and Libraries Journal, 26(2), 91-108. doi: 10.1111/j.14711842.2009.00848.x

Griffiths, D., y García-Peñalvo, F. J. (2016). Informal learning recognition and management. Computers in Human Behavior, 55A, 501-503. doi: 10.1016/j. chb.2015.10.019

Gutiérrez Esparza, G., Padilla Díaz, A., CanulReich, J., De-Luna, C. A., y Ponce, J. (2016). Proposal of a Sentiment Analysis Model in Tweets for Improvement of the Teaching -Learning Process in the Classroom Using a Corpus of Subjectivity. International Journal of Combinatorial Optimization Problems and Informatics, $7(2), 22-34$.

Herrera-Bernal, J. A., Ramírez-Hernández, D. C., y Ramírez-Montoya, M. S. (2016). Applied competences for students by using m-learning devices in Higher Education: Knowledge, skills and attitudes. En. L. Briz-Ponce, J. A., Juanes-Méndez, F. J. García-Peñalvo (Eds.), Handbook of Research on Mobile Devices and Applications in Higher Education Settings (453-476). Hershey, PA: IGI Global.

Humante-Ramos, P. R., García-Peñalvo, F. J., y Conde-González, M. Á. (2016). PLEs in Mobile Contexts: New Ways to Personalize Learning. IEEE Revista Iberoamericana de Tecnologias del Aprendizaje, 11(4), 220-226. doi: 10.1109/ RITA.2016.2619121.

International Telecommunication Union. (2016). Measuring the Information. Society Report. Geneva, Switzerland: International Telecommunication Union. Johnson, L., Adams, S., y Cummins, M. (2012). The NMC Horizon Report: 2012 Higher Education Edition. Austin, Texas: The New Media Consortium.

Johnson, L., Adams, S., Cummins, M., Estrada, V., Freeman, A., y Ludgate, H. (2013). NMC Horizon Report: 2013 Higher Education Edition. Austin, Texas: The New Media Consortium.

Joo-Nagata, J., García-Bermejo Giner, J., y Martínez Abad, F. (2017). Augmented Reality in Pedestrian Navigation applied in a context of Mobile Learning: Resources for enhanced comprehension of Science, Technology, Engineering and Mathematics. International Journal of Engineering Education, 33(2B), 768-780. Joo-Nagata, J., García-Bermejo Giner, J., y Muñoz Rodríguez, J. (2015). Herramientas Geomáticas utilizadas en Educación: situación actual y su relación con procesos educativos. Enseñanza \& Teaching. Revista Interuniversitaria de 
Didáctica, 33(1), 25-56. doi: 10.14201/ et20153312556.

Joo-Nagata, J., Martínez Abad, F., GarcíaBermejo Giner, J., y García-Peñalvo, F. J. (2017). Augmented reality and pedestrian navigation through its implementation in m-learning and e-learning: Evaluation of an educational program in Chile. Computers \& Education, 111, 1-17. doi: 10.1016/j.compedu.2017.04.003.

Karapanos, E., Teixeira, P., \& Gouveia, R. (2016). Need fulfillment and experiences on social media: A case on Facebook and WhatsApp. Computers in Human Behavior, 55, Part B, 888-897. doi: http:// dx.doi.org/10.1016/j.chb.2015.10.015

Keller, J. M. (2010). Motivational design for learning and performance. New York: Springer Science+Business.

Kitchenham, B., \& Charters, S. (2007). Guidelines for performing Systematic Literature Reviews in Software Engineering. Version 2.3 (EBSE2007-01). Recuperado de http:// www.elsevier.com/data/promis misc/525444systematicreviewsguide.pdf

Lai, C.-L., y Hwang, G.-J. (2014). Effects of mobile learning time on students' conception of collaboration, communication, complex problemsolving, meta-cognitive awareness and creativity. International Journal of Mobile Learning and Organisation, 8(3-4), 276291. doi: 10.1504/IJMLO.2014.067029.

Lewin, K. (1947). Frontiers in group dynamics: Concept, method, and reality in social sciences, social equilibria, and social change. Human Relations, 1(1), 5-41. doi: 10.1177/001872674700100103. Observatorio de Innovación Tecnológica del Tecnológico de Monterrey. (2014). Aprendizaje invertido. Recuperado de edutrendsaprendizajeinvertido

Padrón Moctezuma, M. S., Ochoa-Vásquez, M. Á., y Ramírez-Montoya, M. S. (2016). Net Generation Features that Enhance Mobile Learning. En. L. Briz-Ponce, J. A., Juanes-Méndez, F. J. García-Peñalvo (Eds.), Handbook of Research on Mobile Devices and Applications in Higher Education Settings (92-117). Hershey, PA: IGI Global.

Palomo-Duarte, M., Berns, A., Cejas, A., Dodero, J. M., y Caballero, J. A. (2016). Assessing foreign language learning through mobile game-based learning environments. International Journal of Human Capital and Information Technology Professionals (IJHCITP), 7(2), 53-67. doi: 10.4018/ IJHCITP.2016040104.

Ramos, A. I., Herrera-Bernal, J.A., y RamírezMontoya, M. S. (2010). Desarrollo de habilidades cognitivas con aprendizaje móvil: un estudio de casos. Comunicar: Revista Científica Iberoamericana de Comunicación y Educación, XVII(34), 201-209. doi: 10.3916/C34-2010-03-20.

Ramírez-Montoya, M. S. (2009). Recursos tecnológicos para el aprendizaje móvil (mlearning) y su relación con los ambientes de educación a distancia: Implementaciones e investigaciones. Revista Iberoamericana de Educación a Distancia, 12(2), 57-82.

Ramírez-Montoya, M. S. (2017). Training educational researchers in Science and Mathematics: A case study through a binational workshop Mexico-UK. En M. S. Ramírez-Montoya (Ed.), Handbook of Research on Driving STEM Learning with Educational Technologies (1-21). EEUU: IGI Global. 
Ramírez-Montoya, M. S., y Burgos, J. V. (2012). Proyecto macro de la experiencia de investigación Recursos educativos abiertos y móviles para la formación de investigadores educativos: redes académicas, repositorio DAR y REA producidos. En M.S. Ramírez-Montoya y J. V. Burgos (Eds.), Recursos educativos abiertos y móviles para la formación de investigadores: Investigaciones $y$ experiencias prácticas (9-29). México: Lulú editorial digital.

Ramírez-Montoya, M. S., y RamírezHernández, D. C. (2016). Inverted Learning Environments with Technology, Innovation and Flexibility: Student experiences and meanings. Journal of Information Technology Research, 9(1), 18-33. doi: 10.4018/JITR.2016010102.

Ruiz-Rube, I., Miguel Mota, J., Person, T., Berns, A., y Dodero Beardo, J. M. (2016). Autoría y analítica de aplicaciones móviles educativas multimodales. En F. J. García-Peñalvo y J. A. Mendes (Eds.), XVIII Simposio Internacional de Informática Educativa, SIIE 2016 (289294). Salamanca, España: Ediciones Universidad de Salamanca.

Sánchez, A., Redondo, E., y Fonseca, D. (2012). Developing an augmented reality application in the framework of architecture degree. Proceedings of the 2012 ACM workshop on User experience in e-learning and augmented technologies in education, UXeLATE '12, Nara, Japan - November o2 - o2, 2012 (pp. 37-42). New York, NY, USA: ACM.

Sánchez-Prieto, J. C., Olmos-Migueláñez, S., y García-Peñalvo, F. J. (2013). Mobile Learning: Tendencies and Lines of Research. En F. J. García-Peñalvo (Ed.), Proceedings of the First International
Conference on Technological Ecosystems for Enhancing Multiculturality (TEEM'13) (Salamanca, Spain, November 14-15, 2013) (pp. 473-480). New York, NY, USA: ACM.

Sánchez-Prieto, J. C., Olmos-Migueláñez, S., y García-Peñalvo, F. J. (2014). Understanding mobile learning: devices, pedagogical implications and research lines. Education in the Knowledge Society, 15(1), 20-42.

Sánchez-Prieto, J. C., Olmos-Migueláñez, S., y García-Peñalvo, F. J. (2016a). Assessment of Pre-Primary Education Pre-Service Teachers Dispositional Resistance to Change Using RCS. En F. J. García-Peñalvo (Ed.), Proceedings of the Fourth International Conference on Technological Ecosystems for Enhancing Multiculturality (TEEM'16) (Salamanca, Spain, November 2-4, 2016) (pp. 211216). New York, NY, USA: ACM.

Sánchez-Prieto, J. C., Olmos-Migueláñez, S., y García-Peñalvo, F. J. (2016b). Enjoyment, Resistance to Change and mLearning Acceptance Among Pre-Service Teachers. En F. J. García-Peñalvo (Ed.), Proceedings of the Fourth International Conference on Technological Ecosystems for Enhancing Multiculturality (TEEM'16) (Salamanca, Spain, November 2-4, 2016) (pp. 691697). New York, NY, USA: ACM.

Sánchez-Prieto, J. C., Olmos-Migueláñez, S., y García-Peñalvo, F. J. (2016c). Subjective Norm and Behavioral Intention to Use Mobile Technologies. En F. J. GarcíaPeñalvo y A. J. Mendes (Eds.), 2016 International Symposium on Computers in Education (SIIE 16) (Salamanca, Spain. September 13-15, 2016). USA: IEEE. 
Sánchez-Prieto, J. C., Olmos-Migueláñez, S., y García-Peñalvo, F. J. (2017a). MLearning and pre-service teachers: An assessment of the behavioral intention using an expanded TAM model. Computers in Human Behavior, In Press. doi: http:// dx.doi.org/10.1016/j.chb.2016.09.061

Sánchez-Prieto, J. C., Olmos-Migueláñez, S., y García-Peñalvo, F. J. (2017c). ¿Utilizarán los futuros docentes las tecnologías móviles? Validación de una propuesta de modelo TAM extendido. RED. Revista de Educación a Distancia, 52, Artículo 5. doi: http:// dx.doi.org/10.6018/red $/ 52 / 5$

Sánchez-Prieto, J. C., Olmos-Migueláñez, S., García-Peñalvo, F. J., y TorrecillaSánchez, E. M. (2016). Las tabletas digitales en educación formal: Características principales y posibilidades pedagógicas. En A. I. Callejas Albiñana, J. V. Salido López y Ó. Jerez García (Eds.), Competencia Digital y Tratamiento de la Información. Aprender en el Siglo XXI.IV Congreso Internacional de Competencias Básicas, Ciudad Real, 9, 10 y 11 de abril de 2014 (pp. 269-280). Cuenca: Ediciones de la Universidad de Castilla-La Mancha.

Sandoval, E. A., García, R., y RamírezMontoya, M. S. (2012). Competencias tecnológicas y de contenido necesarias para capacitar en la producción de recursos de aprendizaje móvil. Edutec-e: Revista Electrónica de Tecnología Educativa, 39.
Sein-Echaluce Lacleta, M. L., Fidalgo Blanco, Á., y García-Peñalvo, F. J. (2015). Metodología de enseñanza inversa apoyada en b-learning y gestión del conocimiento. En Á. Fidalgo Blanco, M. L. Sein-Echaluce Lacleta, y F. J. García-Peñalvo (Eds.), La Sociedad del Aprendizaje. Actas del III Congreso Internacional sobre Aprendizaje, Innovación y Competitividad. CINAIC 2015 (pp. 464-468). Madrid, Spain: Fundación General de la Universidad Politécnica de Madrid.

Sung, Y., Chang, K., y Liu, T. (2016). The effects of integrating mobile devices with teaching and learning on students' learning performance: A meta-analysis and research synthesis. Computers \& Education, 94, 252-275. doi: 10.1016/j. compedu.2015.11.008.

Telefónica. (2012). Universidad 2020: Papel de las TIC en el nuevo entorno socioeconómico. Barcelona: Ariel.

Tourón, J., y Santiago, R. (2015). El modelo Flipped Learning y el desarrollo del talento en la escuela. Revista de Educación, 368, 196-231. doi: 10.4438/1988-592X-RE2015-368-288.

Wilson, S., Liber, O., Johnson, M., Beauvoir, P., Sharples, P., \& Milligan, C. (2007). Personal Learning Environments: Challenging the dominant design of educational systems Journal of e-Learning and Knowledge Society, 3(3), 27-38.

NOTA. Las citas referidas a autores que han publicado su artículo en el presente número de RIED, pueden consultarlas directamente en el volumen citado 20(2) de RIED. 


\section{PERFIL ACADÉMICO Y PROFESIONAL DE LOS AUTORES}

María Soledad Ramírez-Montoya. Doctora en Filosofía y Ciencias de la Educación por la Universidad de Salamanca (España). Decana asociada de posgrado e investigadora titular de la Escuela de Humanidades y Educación del Tecnológico de Monterrey (ITESM, México). Directora de las Cátedras UNESCO: "Movimiento educativo abierto para América Latina”, de la cátedra OER Latin America del International Council for Open of Distance Education (ICDE, coordinadora del grupo de investigación del Grupo de Investigación e Innovación en Educación (GIIE) del ITESM y profesora del doctorado Formación en la Sociedad del Conocimiento de la Universidad de Salamanca.

E-mail: solramirez@itesm.mx

\section{DIRECCIÓN DE LA AUTORA}

Escuela de Humanidades y Educación

Tecnológico de Monterrey

Avda. Garza Sada 2501 sur; col. Tecnológico

Monterrey, N. L. México; CP64849

Francisco José García-Peñalvo. Ingeniero en Informática y Doctor por la Universidad de Salamanca (España), institución donde se desempeña como profesor y director del Grupo GRIAL. Ha dirigido y participado en más de 50 proyectos de innovación e investigación. Ha publicado más de 200 artículos en revistas y conferencias internacionales y ha sido editor invitado en varios números especiales en revistas internacionales de alto prestigio. Además es editor en jefe de las revistas Education in the Knowledge Society y Journal of Information Technology Research. Es profesor distinguido de la Escuela de Humanidades y Educación del Instituto Tecnológico de Monterrey en México.

E-mail: fgarcia@usal.es

\section{DIRECCIÓN DEL AUTOR}

Departamento de Informática y Automática

Instituto Universitario de Ciencias de la Educación (IUCE), Grupo de Investigación GRIAL

Universidad de Salamanca

Paseo de Canalejas 169, 37008

Salamanca (España) 
Fecha de recepción del artículo: 01/05/2017

Fecha de aceptación del artículo: 17/05/2017

\section{Como citar este artículo:}

Ramírez-Montoya, M. S., y García-Peñalvo, F. J. (2017). La integración efectiva del dispositivo móvil en la educación y en el aprendizaje. RIED. Revista Iberoamericana de Educación a Distancia, 20(2), pp. 29-47. doi: http://dx.doi. org/10.5944/ried.20.2.18884 